\title{
Delayed treatment-free response after romiplostim discontinuation in pediatric chronic immune thrombocytopenia
}

\author{
Hyun Ji Lim ${ }^{1}$, Young Tae Lim ${ }^{1}$, Jeong Ok Hah ${ }^{2}$, Jae Min Lee ${ }^{3}$ \\ ${ }^{1}$ Department of Pediatrics, Yeungnam University Hospital, Daegu, Korea \\ ${ }^{2}$ Department of Pediatrics, Daegu Fatima Hospital, Daegu, Korea \\ ${ }^{3}$ Department of Pediatrics, Yeungnam University College of Medicine, Daegu, Korea
}

Received: June 18, 2020

Revised: July 13, 2020

Accepted: July 18, 2020

Corresponding author:

Jae Min Lee, MD, PhD

Department of Pediatrics,

Yeungnam University College of

Medicine, 170 Hyeonchung-ro,

Nam-gu, Daegu 42415, Korea

Tel: +82-53-620-3536

Fax: +82-53-629-2252

E-mail:mopic@hanmail.net
We report the case of a 16-month-old patient with chronic immune thrombocytopenia (ITP) patient who experienced delayed treatment-free response (TFR) after romiplostim treatment. He received intravenous immunoglobulin every month to maintain a platelet count above $20,000 / \mu \mathrm{L}$ for 2 years. Thereafter, he received rituximab and cyclosporine as second-line therapy, with no response, followed by romiplostim. After 4 weeks of treatment, the platelet count was maintained above 50,000/ $\mu \mathrm{L}$. Following 7 months of treatment, he discontinued romiplostim, and the platelet count decreased. His platelet counts remained above $50,000 / \mu \mathrm{L}$, without any bleeding symptoms, 2 years after romiplostim discontinuation. This is the first report of TFR after romiplostim treatment in pediatric chronic ITP.

Keywords: Child; Immune thrombocytopenia; Idiopathic thrombocytopenic purpura; Romiplostim; Treatment-free response

\section{Introduction}

Immune thrombocytopenia (ITP) is the most common cause of thrombocytopenia in children [1]. Chronic ITP lasts for over 12 months after the initial diagnosis. Most patients with chronic ITP cannot achieve remission with conventional treatments (intravenous immunoglobulin [IVIG], corticosteroid, and anti-D immunoglobulin) or experience relapse after remission [2]. Thrombopoietin receptor agonists (TPO-RAs) such as romiplostim and eltrombopag are promising agents against chronic ITP in children and adults [3]. Romiplostim stimulates TPO receptors to increase platelet production in chronic ITP. Romiplostim is approved for use in adults and children [3]. Some patients experience a treatment-free response (TFR) after treatment with TPO-RA [4]. Here we report the case of a delayed TFR after romiplostim treat- ment in a pediatric patient with chronic ITP.

\section{Case}

This study was approved by the Institutional Review Board (IRB) of the Yeungnam University Medical Center (IRB No: 2019-12024). Written informed consent was obtained from the parent/ guardian for publication of this case report and accompanying images.

A 16-month-old boy presented with petechiae, bruises, and ecchymoses of the trunk and extremities. He was diagnosed with acute ITP and was treated with IVIG. He relapsed within a month and was tested for a secondary cause of ITP. The tests were performed to differentiate between viral infections and autoimmune diseases, which can cause secondary ITP. The laboratory findings

Copyright (C) 2021 Yeungnam University College of Medicine

This is an Open Access article distributed under the terms of the Creative Commons Attribution Non-Commercial License (http://creativecommons.org/licenses/by-nc/4.0/) which permits unrestricted non-commercial use, distribution, and reproduction in any medium, provided the original work is properly cited. 
were as follows: white blood cell count, $17,980 / \mu \mathrm{L}$ ( $58 \%$ neutrophils); hemoglobin level, $14 \mathrm{~g} / \mathrm{dL}$; and platelet count, $3.000 / \mu \mathrm{L}$. Kidney and liver function test results were unremarkable. Other laboratory findings were as follows: C-reactive protein level, 0.563 $\mathrm{mg} / \mathrm{dL}$ (range, $<0.5 \mathrm{mg} / \mathrm{dL}$ ); erythrocyte sedimentation rate, 22 $\mathrm{mm} /$ hour (range, 0-20 mm/hour); C3 level, $159.4 \mathrm{mg} / \mathrm{dL}$ (range, $90-180 \mathrm{mg} / \mathrm{dL}$ ); C4 level, $28.2 \mathrm{mg} / \mathrm{dL}$ (range, 10-40 $\mathrm{mg} / \mathrm{dL}$ ); CH50 level, $40.8 \mathrm{U} / \mathrm{mL}$ (range, $23-46 \mathrm{U} / \mathrm{mL}$ ); antinuclear antibody, negative; anti-Smith antibody, negative; antiplatelet antibody, negative; and venereal disease research laboratory test, non-reactive. In addition, the bone marrow showed increased numbers of megakaryocytes and non-platelet budding micro-megakaryocytes as well as increased cellularity. Therefore, he was diagnosed with primary ITP.

He was treated with IVIG $(1 \mathrm{~g} / \mathrm{kg} /$ day for 2 days $)$ every 4 to 6 weeks for 2 years to maintain the platelet count above $20,000 / \mu \mathrm{L}$. His platelet count showed a pattern of a temporary increase to over 100,000/ $\mu \mathrm{L}$ after IVIG administration and then decreased to less than $10,000 / \mu \mathrm{L}$ after 2 to 3 weeks. Steroids such as dexamethasone and prednisolone had no effect on the platelet count, which was continuously less than $10,000 / \mu \mathrm{L}$. Systemic bruising, petechiae, and mucocutaneous bleeding occurred repeatedly when the platelet levels dropped below $20,000 / \mu \mathrm{L}$. Two years after diagnosis, he was treated with rituximab $\left(375 \mathrm{mg} / \mathrm{m}^{2} /\right.$ day for 4 weeks) and cyclosporine as second-line therapy, but he showed no response. The patient was treated with romiplostim (initial dose, $1 \mu \mathrm{g} / \mathrm{kg} /$ week) at 49 months (i.e., 33 months after diagno- sis); the romiplostim dose was later increased to $10 \mu \mathrm{g} / \mathrm{kg} /$ week. Romiplostim was purchased through the Korea Orphan \& Essential Drug Center (KOEDC) (Seoul, Korea). After 4 weeks of treatment, his platelet counts increased (Fig. 1). During the 7 months of romiplostim treatment, he showed no bleeding symptoms and required no additional IVIG or steroid treatment. No adverse effects of romiplostim such as headache, skeletal pain, abdominal pain, or thromboembolism were observed. After 7 months of romiplostim therapy, he discontinued romiplostim due to the economic burden, and his platelet count decreased again while he continued IVIG treatment. However, the frequency of annualized IVIG treatment gradually decreased after romiplostim treatment (Fig. 2).

Two years after romiplostim discontinuation, his platelet count remained above $50,000 / \mu \mathrm{L}$, and he achieved TFR for over 36 months with no treatment. In addition, no bleeding symptoms were observed.

\section{Discussion}

ITP is an autoimmune-mediated acquired bleeding disorder characterized by isolated thrombocytopenia, which is defined as a peripheral blood platelet count below $100 \times 10^{3} / \mu \mathrm{L}$, with no obvious initiating and/or underlying cause. ITP affects patients of all ages, genders, and races; age-specific incidence is the greatest in children and the elderly [5].

The mechanism underlying platelet count reduction in ITP

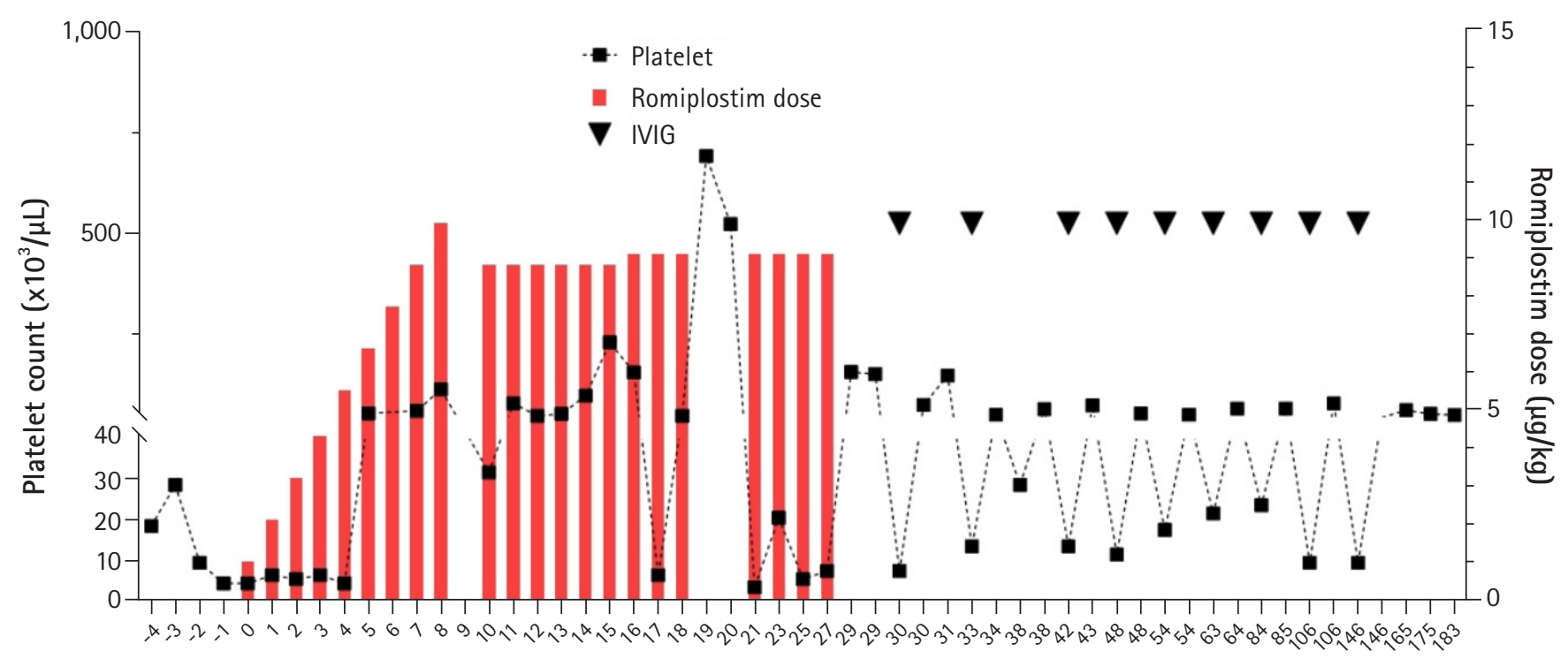

Time after romiplostim administration (wk)

Fig. 1. Changes in the platelet count during romiplostim treatment. IVIG, intravenous immunoglobulin. 


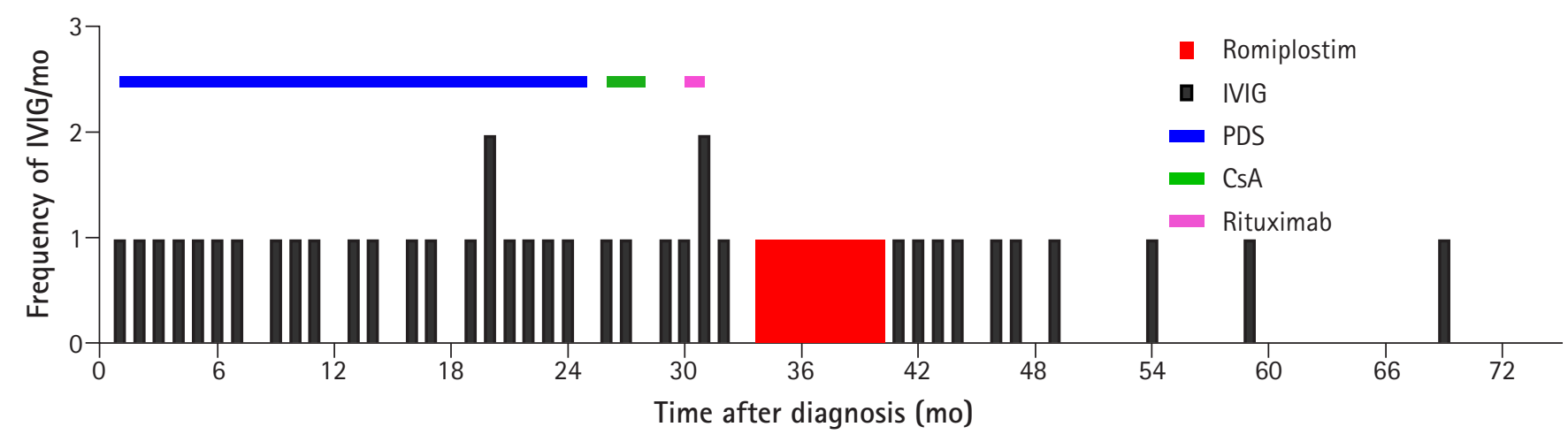

Fig. 2. Treatment course in a patient with chronic immune thrombocytopenia. The frequency of intravenous immunoglobulin (IVIG) administration was reduced after romiplostim treatment. Treatment included prednisone (PDS), IVIG, rituximab, oral cyclosporine (CsA), and romiplostim.

was previously considered to be due to increased platelet destruction by autoantibodies. However, it has recently been found that platelet count reduction in ITP occurs via more complicated mechanisms related to impaired platelet production and $\mathrm{T}$ cell-mediated effects $[6,7]$. ITP usually presents with bleeding, fatigue, thrombosis, and symptoms associated with disorders causing secondary ITP, such as systemic lupus, hepatitis $\mathrm{C}$, infection, and lymphoid malignancy. Primary ITP is diagnosed by exclusion.

TPO is a 94-kDa protein primarily synthesized in the liver and secreted into circulation; it has no storage form. TPO and TPO mimetics reverse the apoptotic effects of antiplatelet/megakaryocyte antibodies (or local T cell inhibition) on late megakaryocyte progenitors, but not on the late megakaryocytes themselves because it takes 5 to 7 days before the platelet count increases in response to a TPO-RA [8].

The efficacy of romiplostim in 125 adult patients with ITP, including 63 splenectomized and 62 non-splenectomized patients, was assessed in a double-blind randomized controlled trial. The overall platelet response rate was $88 \%$ in non-splenectomized patients and $79 \%$ in splenectomized patients treated with romiplostim. In addition, $87 \%$ of patients who received romiplostim reduced or discontinued concurrent therapy. Thus, the study shows that stimulation of platelet production by romiplostim may provide a new therapeutic option for patients with ITP [8].

In a phase 3, randomized, double-blind, and placebo-controlled study of 62 children under 18 years treated for ITP, romiplostim increased platelet counts and improved health-related quality of life in children with primary ITP $[4,9]$. In a study of long-term treatment with romiplostim in children with chronic ITP, romiplostim increased and maintained platelet counts without significant toxicity for $\geq 6$ months [10]. TFR was maintained in $23 \%$ of children for $\geq 6$ months with no medication, including romiplos- tim. In that study, the factors predictive of spontaneous TFR in children with ITP included a higher platelet count at diagnosis ( $>60,000 / \mu \mathrm{L}$ ), younger age, recent onset ( $<2$ weeks) of bleeding symptoms, decreased bleeding in the first 6 months, and highgrade bleeding at diagnosis. In a multicenter Spanish adult study, receiving romiplostim as the first TPO-RA was positively associated with the likelihood of achieving TFR, while switching the TPO-RA negatively predicted TFR [11].

Our patient was diagnosed at a very young age and showed a good response to romiplostim; therefore, romiplostim was not switched to any other TPO-RA. Although his platelet levels decreased after romiplostim discontinuation, a delayed TFR was observed.

Although eltrombopag, another oral TPO, may be a better option when considering patient comfort, it was not recommended for use in children in Korea at the time. Romiplostim was supplied by the KOEDC; therefore, we used romiplostim first.

In conclusion, romiplostim is a potential treatment option for chronic ITP in children when considering the long-term complications of splenectomy, such as infection and thromboembolism after splenectomy. The possibility of TFR is high with romiplostim; therefore, romiplostim should be considered when choosing an alternative therapy in children with chronic ITP. Further studies with long-term follow-up are required to determine the efficacy of romiplostim and TPO-RA-associated TFR.

\section{Acknowledgments}

\section{Conflicts of interest}

No potential conflict of interest relevant to this article was reported. 


\section{Funding}

This work was supported by 2019 Yeungnam University Research Grant.

\section{Author contributions}

Conceptualization: YTL, JOH, JML; Formal analysis and Supervision: JOH, JML; Funding acquisition: JML; Investigation: YTL; Writing-original draft: HJL; Writing-review \& editing: JML.

\section{ORCID}

Hyun Ji Lim, https:/ / orcid.org/0000-0003-4000-9205

Young Tae Lim, https://orcid.org/0000-0001-9926-0467

Jeong Ok Hah, https://orcid.org/0000-0002-4072-7867

Jae Min Lee, https:/ / orcid.org/0000-0001-6822-1051

\section{References}

1. Kim JY. Diagnostic approach of childhood immune thrombocytopenia. Clin Pediatr Hematol Oncol 2018;25:10-6.

2. Kim YK, Lee SS, Jeong SH, Ahn JS, Yang DH, Lee JJ, et al. Efficacy and safety of eltrombopag in adult refractory immune thrombocytopenia. Blood Res 2015;50:19-25.

3. Neunert CE, Rose MJ. Romiplostim for the management of pediatric immune thrombocytopenia: drug development and current practice. Blood Adv 2019;3:1907-15.

4. Tarantino MD, Bussel JB, Blanchette VS, Despotovic J, Bennett C, Raj A, et al. Romiplostim in children with immune thrombo- cytopenia: a phase 3, randomised, double-blind, placebo-controlled study. Lancet 2016;388:45-54.

5. Franchini M, Veneri D, Lippi G. Thrombocytopenia and infections. Expert Rev Hematol 2017;10:99-106.

6. Thota S, Kistangari G, Daw H, Spiro T. Immune thrombocytopenia in adults: an update. Cleve Clin J Med 2012;79:641-50.

7. Kistangari G, McCrae KR. Immune thrombocytopenia. Hematol Oncol Clin North Am 2013;27:495-520.

8. Kuter DJ, Bussel JB, Lyons RM, Pullarkat V, Gernsheimer TB, Senecal FM, et al. Efficacy of romiplostim in patients with chronic immune thrombocytopenic purpura: a double-blind randomised controlled trial. Lancet 2008;371:395-403.

9. Mathias SD, Li X, Eisen M, Carpenter N, Crosby RD, Blanchette VS. A phase 3, randomized, double-blind, placebo-controlled study to determine the effect of romiplostim on health-related quality of life in children with primary immune thrombocytopenia and associated burden in their parents. Pediatr Blood Cancer 2016;63:1232-7.

10. Tarantino MD, Bussel JB, Blanchette VS, Beam D, Roy J, Despotovic J, et al. Long-term treatment with romiplostim and treatment-free platelet responses in children with chronic immune thrombocytopenia. Haematologica 2019; 104:2283-91.

11. Lozano ML, Mingot-Castellano ME, Perera MM, Jarque I, Campos-Alvarez RM, González-López TJ, et al. Deciphering predictive factors for choice of thrombopoietin receptor agonist, treatment free responses, and thrombotic events in immune thrombocytopenia. Sci Rep 2019;9:16680. 